EPJ manuscript No.

(will be inserted by the editor)

\title{
Size dependence of multipolar plasmon resonance frequencies and damping rates in simple metal spherical nanoparticles
}

\author{
A. Derkachova and K. Kolwas \\ Institute of Physics, Polish Academy of Sciences, \\ Al. Lotników 32/46, 02-668 Warsaw, Poland
}

\begin{abstract}
Multipolar plasmon oscillation frequencies and corresponding damping rates for nanospheres formed of the simplest free-electron metals are studied. The possibility of controlling plasmon features by choosing the size and dielectric properties of the sphere surroundings is discussed. Optical properties of the studied metals are described within the Drude-Sommerfeld model of the dielectric function with effective parameters acounting for the contribution of conduction electrons and of interband transitions. No approximation is made in respect of the size of a particle; plasmon size characteristics are described rigorously. The results of our experiment on sodium nanodroplets 1 are compared with the oscillation frequency size dependence of dipole and quadrupole plasmon.
\end{abstract}

Key words. Metal nanoparticles, surface plasmons, plasmon resonance frequencies, plasmon damping rates, plasmonics, nanophotonics.

\section{Introduction}

The possibility of excitation and observation of surface plasmons in spherical metal particles is a subject of continuously increasing interest. It is connected with a wide range of applications of plasmon excitations in nanotechnology, biophysics, biochemistry etc. The most attractive feature of the surface plasmon resonances is the concentration of electromagnetic field energy near the particle surface. The Surface Enhanced Raman Spectroscopy is based on this phenomenon 2[3. SERS spectroscopic techniques allow spectral studying of single molecules, particles and cells 415. Small noble metal spheres (with size from 20 to 120 nanometers), introduced into an investigated biomaterial, can be used as markers of some specific biomolecules, tissue cancer changes or viruses 6 6]

The frequency dependence of the optical properties of a simple bulk metal (alkali metal) change with free electron density, electron relaxation rates, and the contribution of bound electrons to the polarizability 89 . The simplest model for the dielectric function of bulk metal is the Drude-Sommerfeld model of free electron gas. The optical properties of metal nanospheres, as well as of others nanostructured metal materials, are in addition geometry and (or) size dependent. These futures are caused by the confinement of the electron gas resulting from the presence of metal-dielectric boundary. In particular, optical properties of spherical metal particles are characterized by size dependent discrete eigenfrequencies. These eigenfrequencies can manifest as resonances in the optical response of a sphere to the external electromagnetic field. The complex eigenfrequencies define the plasmon oscillation frequencies and the damping rates of collective surface electron density oscillations which can be excited by the external electromagnetic field. In contrast to the flat metal surface, the curved surface enables the direct optical excitation of surface plasmons. 
In this paper we present a solution of the eigenproblem of nanospheres formed of the simplest free-electron metals. The analysis is concentrated on the influence of size and of material parameters upon the multipolar plasmon features. Optical properties of the studied metals are described within the Drude-Sommerfeld model of the dielectric function with effective parameters accounting for the contribution of interband transition to the dielectric properties of metal. No approximation is made in respect of the size of a particle; plasmon size characteristics are described rigorously. We discuss the role of the material parameters characterizing the electromagnetic properties of nanospheres in controlling of plasmon features. We compare the expected size dependence of plasmon oscillation frequency of dipole and quadrupole plasmon with the results of our experiment on sodium nanodroplets [1].

\section{Eigenvalue problem for a metal sphere}

The eigenvalue problem is formulated in absence of external fields. The eigenvalues result from the condition that the harmonic solutions of Maxwell equations exist in both; the metal sphere and its dielectric surroundings. The discrete complex frequencies of electromagnetic fields result from the continuity relations (in spherical coordinates) at the sphere boundary of the transverse magnetic solutions of Maxwell equations (TM polarization). These fields are coupled to the collective surface electron density oscillations at the sphere surface, that are called surface plasmons. The eigenfrequencies problem was presented in more detail e.g. in [10] for the flat metal-dielectric interface and e.g. in [1011 for the spherical interface. At the flat boundary, the surface plasmon dispersion relation can be obtained in a simple analytical form. The wave vector of a surface plasmon wave $k_{s p}$ [10]:

$$
k_{s p}=\frac{\omega}{c} \sqrt{\frac{\varepsilon_{m}(\omega) \varepsilon_{d}(\omega)}{\varepsilon_{m}(\omega)+\varepsilon_{d}(\omega)}}
$$

where $\varepsilon_{m}(\omega)$ and $\varepsilon_{d}(\omega)$ are the dielectric function of the metal and of the dielectric surroundings respectively. For free-electron metal described by the relaxation-free Drude dielectric function: $\varepsilon_{m}(\omega)=1-\omega_{p}^{2} / \omega^{2}$, and $\varepsilon_{d}(\omega)=1$, the dispersion relation 1 leads to the well known "surface-plasmon frequency" at $\omega=\omega_{p} / \sqrt{2}[10$.

However, in the case of a spherical boundary, the plasmon dispersion relation results from solution of the dispersion equation in complex form:

$$
\sqrt{\varepsilon_{\text {in }}} \xi_{l}^{\prime}\left(k_{\text {out }} R\right) \psi_{l}\left(k_{\text {in }} R\right)-\sqrt{\varepsilon_{\text {out }}} \xi_{l}\left(k_{\text {out }} R\right) \psi_{l}^{\prime}\left(k_{\text {in }} R\right)=0,
$$

with $l=1,2,3 \ldots$ where the wave numbers $k_{i n}$, and $k_{o u t}$ are equal to:

$$
\begin{aligned}
k_{\text {in }} & =\frac{\omega}{c} \sqrt{\varepsilon_{\text {in }}(\omega)}, \\
k_{\text {out }} & =\frac{\omega}{c} \sqrt{\varepsilon_{\text {out }}(\omega)} .
\end{aligned}
$$

$\varepsilon_{\text {in }}$ and $\varepsilon_{\text {out }}$ are dielectric functions of the investigated metal sphere and of the dielectric environment respectively, and define the corresponding refraction coefficients: $n_{i n}=\sqrt{\varepsilon_{i n}}$ and $n_{\text {out }}=\sqrt{\varepsilon_{\text {out }}} \cdot \psi_{l}(z)$ and $\xi_{l}(z)$ are Riccati-Bessel spherical functions which can be expressed by the Bessel $J_{l+\frac{1}{2}}(z)$, Hankel $H_{l+\frac{1}{2}}^{(1)}(z)$ and Neuman $N_{l+\frac{1}{2}}(z)$ cylindrical functions of the half order, defined (e.g. in [12]) as:

$$
\begin{aligned}
& \psi_{l}(z)=z \cdot j_{l}(z)=z \sqrt{\frac{\pi}{2 z}} J_{l+\frac{1}{2}}(z), \\
& \xi_{l}(z)=\psi_{l}(z)-i \cdot \chi_{l}(z)=z \cdot h_{l}^{(1)}(z)=z \sqrt{\frac{\pi}{2 z}} H_{l+\frac{1}{2}}^{(1)}(z), \\
& \chi_{l}(z)=z \sqrt{\frac{\pi}{2 z}} N_{l+\frac{1}{2}}(z) .
\end{aligned}
$$


Solutions of the dispersion equations 2 for each $l$ mode exist only for the complex frequencies of the TM (transverse magnetic) polarized electromagnetic field at the sphere boundary, at $r=R$ [10]11:

$$
\Omega_{l}(R)=\omega_{l}^{\prime}(R)+i \cdot \omega_{l}^{\prime \prime}(R),
$$

and can be found numerically for known dielectric functions of the metal sphere $\varepsilon_{i n}(\omega)$ and its dielectric surroundings $\varepsilon_{\text {out }}(\omega) \cdot \omega_{l}^{\prime}(R)$ are the oscillation frequencies of TM electromagnetic field at the surface in mode $l=1,2,3 \ldots \omega_{l}^{\prime \prime}(R)$ are the damping frequencies of these oscillations, and are the convolution of the radiative damping and of electron relaxation processes.

\section{Drude-Sommerfeld model of the dielectric function}

Some of the metal properties, including the optical properties, can be described within the simple free-electron gas Drude-Sommerfeld model of the dielectric function. In the framework of this model, with an external field applied, the conduction electrons move freely between independent collisions occurring at the average rate of $\gamma$. The frequency dependent dielectric function $\varepsilon(\omega)$ predicted by Drude-Sommerfeld model:

$$
\varepsilon(\omega)=\varepsilon_{\infty}-\frac{\omega_{p}^{2}}{\omega^{2}+i \gamma \omega},
$$

includes the contribution of the bound electrons to the polarizability by introducing phenomenological parameter $\varepsilon_{\infty}$. This parameter equals 1 only if the conduction band electrons contribute to the dielectric properties. The plasma frequency $\omega_{p}$ is given by:

$$
\omega_{p}=\sqrt{\frac{N e^{2}}{\varepsilon_{0} m^{*}}},
$$

where $N$ and $m^{*}$ are the density of conduction electrons and the electron effective mass respectively.

In order to solve the dispersion equation 2 with respect to the frequency, we assumed that for the best free-electron metal: $\varepsilon_{i n}(\omega)=\varepsilon(\omega)$ (eq. 9) with the following parameters: $\varepsilon_{\infty}^{N a}=1.06$ [13], $\omega_{p}^{N a}=5.6 \mathrm{eV}[8]$ and $\gamma^{N a}=0.03 \mathrm{eV}$ for sodium, $\varepsilon_{\infty}^{L i}=5.843, \omega_{p}^{L i}=8 \mathrm{eV}$ [8] and $\gamma^{L i}=0.05 \mathrm{eV}$ for lithium, and $\varepsilon_{\infty}^{C s}=1.8, \omega_{p}^{C s}=3.4 \mathrm{eV}\left[8\right.$ and $\gamma^{C s}=0.03 \mathrm{eV}$ for cesium. The dielectric function 9 for sodium (solid line in Fig. 1 a) and b)) reproduces the optical constants $n$ measured for liquid and solid sodium[1415] (open and closed circles on Fig. 1) quite well. However, the dielectric properties for lithium [15] (squares in Fig. 1 a) and b)) and cesium [16] (triangles in Fig. 1 a) and b)) are more complex, and are less satisfactory reproduced by the Drude-Sommerfeld dielectric function in the studied frequency range, as illustrated in Fig. 1 (dotted line for $L i$ and dashed line for $C s$ ).

The proper choice of the parameters entering the dielectric function is crucial to predicting plasmon resonance characteristics in experimental realizations. Let's notice, that the optical constants of metals 14/15/16 were measured in high vacuum conditions and for metals of extremely clean surfaces. However, optical experiments with metal nanoparticles are performed usually under less strict laboratory conditions, for rather contaminated particles. Contamination can be caused by the presence of the atmosphere 1117] and as a result of storing the particles [18 or the bulk metal 17 in some liquids before the experiments. Therefore, the experimental data concerning the plasmon resonance position can be shifted in respect of the predictions assuming "ideal" dielectric properties of a metal. Below we discuss the trends of expected corrections to the plasmon resonance frequencies due to the modifications in parameters $\varepsilon_{\infty}$, $\omega_{p}$ and $\gamma$ entering the metal dielectric function 9. We also demonstrate the importance of the optical properties of environment in determining the position of plasmon resonance of given polarity $l$. 


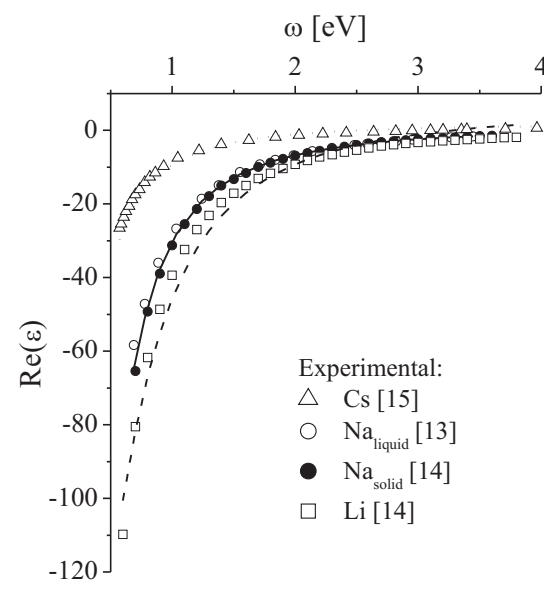

a)

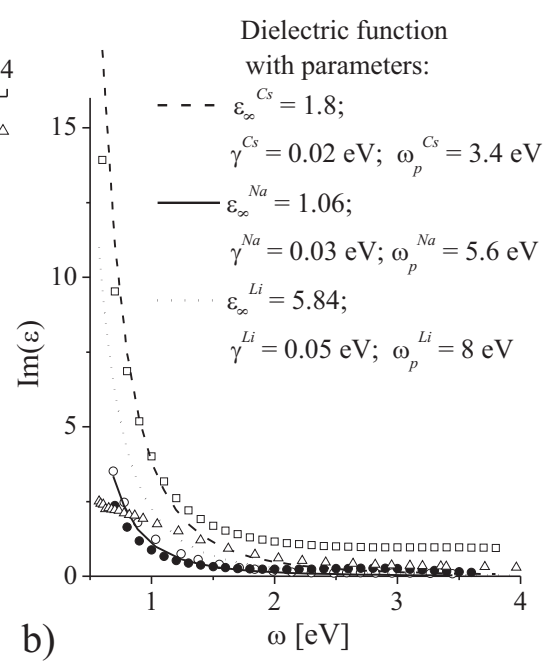

Fig. 1. The real and imaginary part of the dielectric function with the effective parameters $\varepsilon_{\infty}, \gamma$ and $\omega_{p}$ for cesium, sodium and lithium (dashed, solid and dotted line). Triangles, circles and squares mark $\operatorname{Re}\left(n^{2}\right)$ and $\operatorname{Im}\left(n^{2}\right)$ values, resulting from measuring the optical constants $n$ of the corresponding metals [13-15].

\section{Results and discussion}

Figures 2 a)-f) illustrate the multipolar $(l=1,2 \ldots 6)$ plasmon resonance frequencies $\omega_{l}^{\prime}(R)$ and the corresponding damping rates $\omega_{l}^{\prime \prime}(R)$, resulting from solving the dispersion equation 2 with respect to the frequency allowed being complex. We have used the Müller method of secants for finding the numerical solutions of $f(v)=0$ assuming the starting approximated values of the function parameter $v$ in the vicinity of the exact value which may be complex (the "root" function of the Mathcad program). For given $l$, the successive values of $R$ were treated as external parameters and where changed with step $\Delta R \approx 2 \mathrm{~nm}$ up to the final value of $R=200 \mathrm{~nm}$. The starting, approximated values for $\omega_{l}^{\prime}(R)$ entering the root procedure were found from the range between $\omega_{p} / \sqrt{\varepsilon_{\infty}+\varepsilon_{\text {out }}(l+1) / l}$ to $\omega_{p} / \sqrt{2}$ and the negative values of $\omega_{l}^{\prime \prime}$ were assumed.

Plasmon oscillations are always damped (Fig. 2 b), d) and e)) due to radiation and the relaxation processes included in the relaxation rate $\gamma$. The initial increase of the damping rate for given oscillation mode $l$, is followed by a decrease of $\left|\omega_{l}^{\prime \prime}(R)\right|$ for sufficiently large particles, as demonstrated for the dipole plasmon damping rate $(l=1)$. The plasmon damping rate dependence on particle size $\omega_{l}^{\prime \prime}(R)$ is dominated by the radiative damping [1].

Excitation of plasmon resonance in a sphere of given radius $R$ takes place when a frequency $\omega$ of the external electromagnetic wave fits the frequency of a plasmon mode of given multipolarity $l: \omega=\omega_{l}^{\prime}(R)$. For all studied simple-metal spheres (Figures 2 a)-f)), plasmon oscillations can be excited at optical frequencies. For the source of light of broad spectrum (as in experiments using dark-field microscopic techniques reported e.g in [18] or [19]), not only dipole, but also higher multipolar plasmon resonances can be excited. As we have demonstrated for sodium spheres in [11], the highest possible plasmon multipolar resonance frequency $\omega_{0, l}^{\prime}$ and the corresponding damping rate $\omega_{0, l}^{\prime \prime}$ can be attributed to a sphere of a minimum radius $R_{\min , l}$ : that is: $\omega_{0, l}^{\prime}=\omega_{0, l}^{\prime}\left(R_{\min , l}\right), \omega_{0, l}^{\prime \prime}=\omega_{0, l}^{\prime \prime}\left(R_{\min , l}\right) . R_{\min , l}$ being the fast increasing function of the plasmon multipolarity $l$.

For a given particle size, the frequency of plasmon oscillation increases with increasing plasma frequency $\omega_{p}$ (free-electron concentration $N$ ). For example, the dipole plasmon resonance frequency of a particle of $50 \mathrm{~nm}$ radius is smaller for cesium than for sodium, both metals with parameter $\varepsilon_{\infty}$ only slightly differing from 1 . With decreasing size the dipole plasmon oscillation frequencies are slightly modified with respect to the frequency $\omega_{0, l=1}^{\prime}=\omega_{p} / \sqrt{3}$ of so 

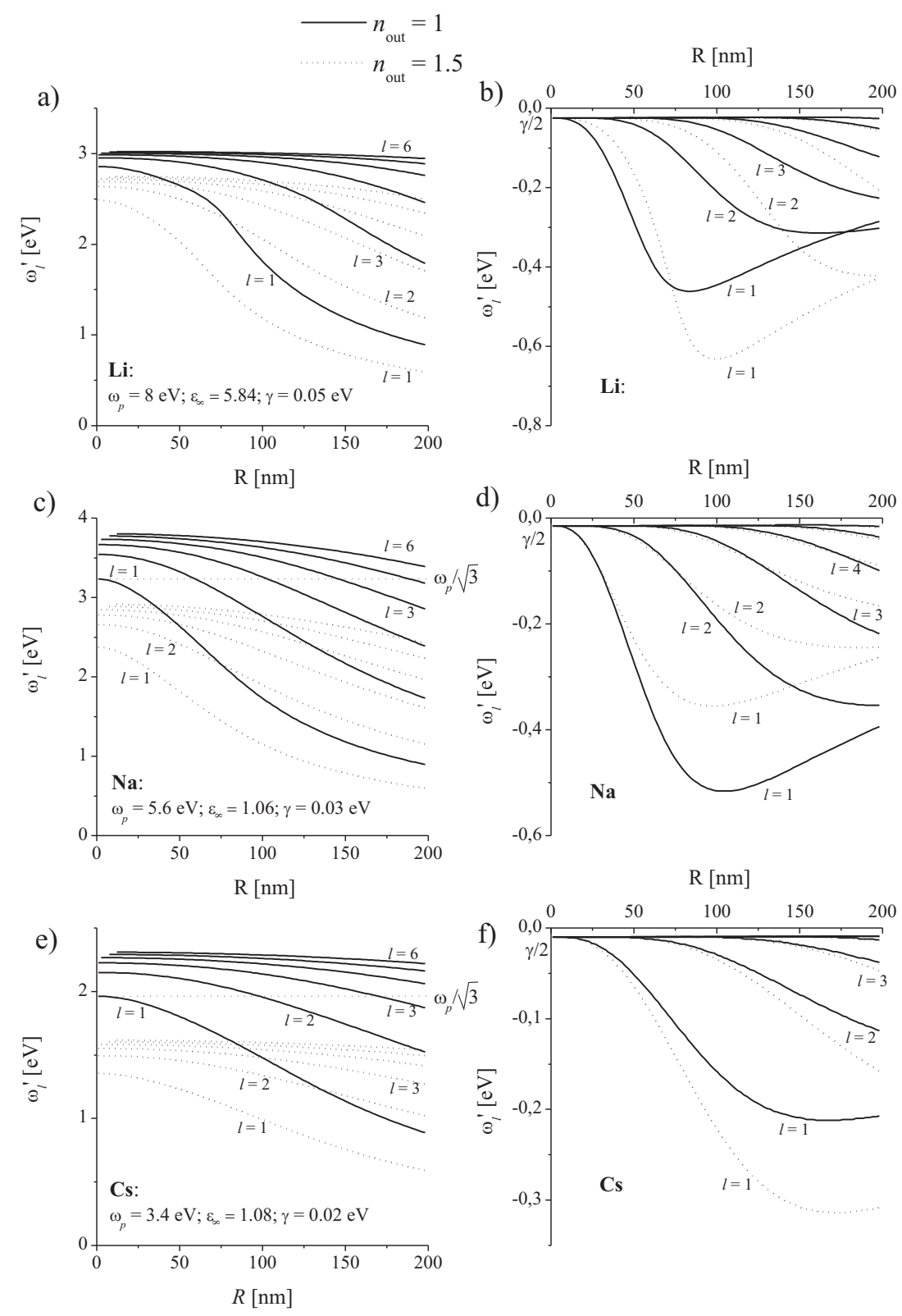

Fig. 2. Multipolar plasmon resonance frequencies $\omega_{l}^{\prime}(R)$ and plasmon oscillation damping rates $\omega_{l}^{\prime \prime}(R)$ as a function of the radius $R$ (rigorous modelling) for lithium, sodium and cesium nanospheres in vacuum $\left(n_{\text {out }}=1\right.$, solid lines $)$, and embedded in glass $\left(n_{\text {out }}=1.5\right.$, short-dashed lines). The first six $(l=1,2 \ldots 6)$ multipolar plasmon characteristics are presented.

called "Mie resonance" 9]. However for lithium, with large value of $\varepsilon_{\infty}$, the dipole plasmon frequency $\omega_{0, l=1}^{\prime}$ is strongly red shifted with respect to the $\omega_{p} / \sqrt{3}$, as for all the higher order plasmon frequency dependence upon size.

As demonstrated in Fig. 2 a)-f) (short-dashed lines), the dielectric properties of the sphere environment can introduce drastic changes to the multipolar plasmon resonance frequency dependence $\omega_{l}^{\prime}(R)$ as well as to the corresponding plasmon damping rates $\omega_{l}^{\prime \prime}(R)$; the proper choice of the refractive index of the environment is the most effective tool (and the easiest in practical application) for controlling plasmon resonance futures. 


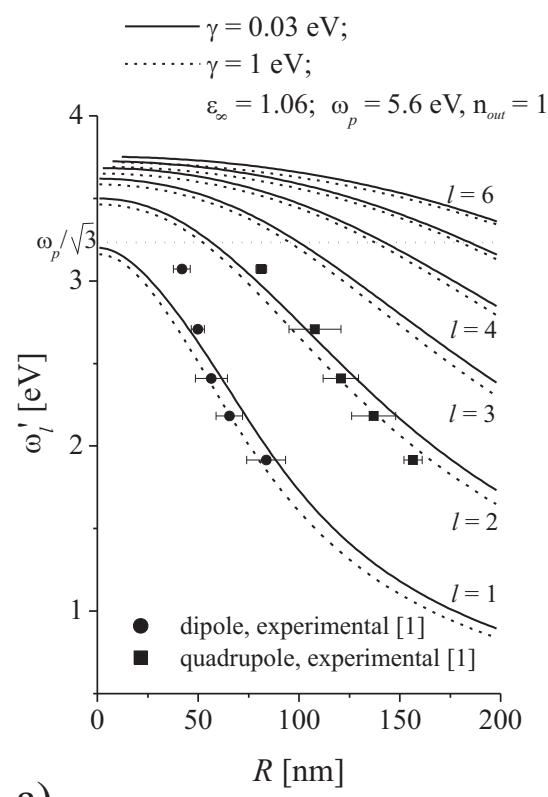

a)

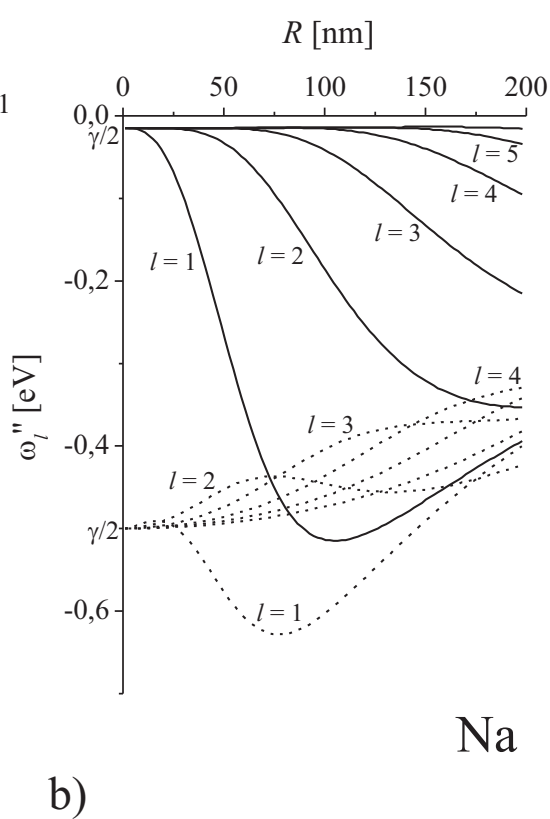

b)

Fig. 3. Multipolar plasmon resonance frequencies $\omega_{l}^{\prime}(R)$ and plasmon oscillation damping rates $\omega_{l}^{\prime \prime}(R)$ as a function of radius $R$ for very clean $(\gamma=0.03 \mathrm{eV}$, solid lines) and contaminated $(\gamma=1 \mathrm{eV}$, shortdashed lines) sodium nanosphere, calculated for $(l=1,2 \ldots 6)$. Circles and squares correspond to the sphere radii allowing to excite the dipole and the quadrupole plasmon resonance with laser light of different wavelength, according to [1].

As we mentioned above, the experimental results concerning the multipolar plasmon resonance frequencies for a particle of a given size can differ from solutions of the eigenproblem with "ideal" dielectric properties assumed. Our experiment on sodium droplets which spontaneously grow after the sodium vapour supersaturation by laser light [117] can serve as an example. Due to the presence of the atmosphere and sodium reactivity, relaxation rate is increased to the value of $\gamma=1 \mathrm{eV}$ [1]17. It red shifts the plasmon resonance frequencies $\omega_{l}^{\prime}(R)$ and introduces important modification to the plasmon damping rates $\omega_{l}^{\prime \prime}(R)$, as illustrated in Fig. 3 a) and b). However, if $\gamma \ll \omega$, electron relaxation causes negligible shift of plasmon resonance frequency, while plasmon damping rates remain dominated by the size dependence of the radiative damping, as demonstrated in 11 for sodium spheres after assuming $\gamma=0$ in the analysis.

The utility value of the elaborated numerical tool for predicting the multipolar plasmon resonance characteristics depends on the quality of reproducing the actual optical properties of a metal by the dielectric function with the effective parameters. It is worth noting however that such fitting can be reduced to the frequency range of interest in a particular plasmon application which corresponds to $1 \mathrm{eV} \div 4 \mathrm{eV}$, as illustrated in Fig.2 a), c) and e) for studied metals.

The elaborated numerical algorithm allows predicting the dependence of plasmon characteristics upon size of any metal spherical particle of known form of the dielectric function $\varepsilon(\omega)$. Such tool can help in tailoring multipolar plasmon resonance properties according to the requirements of particular application by choosing the proper size and the material properties of a nanosphere, as well as the appropriate particle environment.

\section{References}

1. A. Derkachova and K. Kolwas, Proceedings of the SPIE, 5849, (2005) 150-153.

2. K. Kneipp, H. Kneipp, I. Itzkan, R.R. Dasari, and M.S. Feld, J. Phys. C, 14, (2002) R597. 
3. M. Moskovits, Rev. Mod. Phys., 57, (1985) 783.

4. K. Kneipp et al., Phys. Rev. Lett., 78, (1997) 1667.

5. S.M. Nie and S.R. Emory, Science, 275, (1997) 1102.

6. S. Schultz, D.R. Smith, J.J. Mock, and D.A. Schultz, Proc. Natl. Acad. Sci.U.S.A., 97, (2000) 996.

7. S. Schultz, J. Mock, D.R. Smith, and D.A. Schultz, J. of Clinical Ligand Assay, 22, (1999) 214.

8. Ch. Kittel, Introduction to Solid State Physics. (7th Ed., Wiley, 1996).

9. U. Kreibig and M. Vollmer, Optical Properties of Metal Clasters. (Springer, 1995).

10. R. Fuchs and P. Halevi, Basic Concepts and Formalism of Spatial Dispertion in Spatial Dispertion in Solids and Plasmas (North-Holland, 1992).

11. K. Kolwas, A. Derkachova, and S. Demianiuk, Comp. Mat. Sc., 35, (2006) 337.

12. M. Born and E. Wolf, Principles of optics. (Pergamon Press, Oxford, 1975).

13. A.J. Sievers, Phys. Rev. B, 22, (1980) 1600.

14. T. Inagaki, E.T. Arakawa, R.D. Birkhoff, and M.W. Williams, Phys. Rev. B, 13, (1976) 5610.

15. T. Inagaki, L.C. Emerson, E.T. Arakawa, and M.W. Williams, Phys. Rev. B, 13, (1976) 2305.

16. N. V. Smith, Phys. Rev. B, 2, (1970) 2840.

17. S. Demianiuk and K. Kolwas, J. Phys. B, 34, (2001) 1651.

18. C. Sönnichsen and T. Franzl and T. Wilk and G. von Plessen and J. Feldmann, New J. Phys. 4, (2002) 93.1.

19. J. Mock, M. Barbic, D. Smith, D. Schultz, S. Schultz, J. Chem. Phys., 116, (2002) 6755. 